\title{
DEGREES OF IRREDUCIBLE MODULAR CHARACTERS OF BLOCKS WITH CYCLIC DEFECT GROUPS
}

\author{
BY BRUCE ROTHSCHILD ${ }^{1}$
}

Communicated by W. Feit, July 25, 1966

Let $G$ be a finite group of order $g^{\prime} p^{a}, p$ a prime, $\left(g^{\prime}, p\right)=1$. Let $D$ be a cyclic group of order $p^{d}, d \geqq 1$, and let $B$ be a block of $G$ with $D$ as a defect group. We obtain the following theorem:

THEOREM. If $\phi$ is an irreducible modular character of $B$ (with respect to $p)$, then the degree of $\phi$ must be congruent $\left(\bmod p^{a-d+1}\right)$ to one of the following values: $-e N,-(e-1) N, \cdots,-2 N,-N,+N, 2 N, \cdots$, $(e-1) N$, eN, where $N \neq \equiv 0\left(\bmod p^{a-d+1}\right)$, and $e(d e f i n e d$ below) divides $p-1$. In particular, the degree of $\phi$ is not divisible by $p^{a-d+1}$. If $B$ is the principal block, then $N \equiv 1(\bmod p)$.

The theorem is already known for $d=a=1$. We prove the theorem here by considering the tree associated with the block $B$. Brauer [1] describes this tree in discussing blocks with $d=1$. Dade [2] generalizes Brauer's results to arbitrary $d$, and his results enable us to associate the same kind of tree with the block $B$. The tree turns out to have a certain number $e$ (defined below) of edges. The question of whether or not every tree with $e$ edges is associated with some block of a group remains open. We now describe the tree (for details see Dade [2]).

Let $C_{0}$ be the centralizer and $N_{0}$ the normalizer of $D$ in $G$. Let $b_{0}$ be some block of $C_{0}$ such that $b_{0}^{G}=B$, and let $E$ be the subgroup of $N_{0}$ fixing $b_{0}$ under conjugation. Denote by $e$ the index $\left(E: C_{0}\right)$. Then $e$ divides $p-1$. The irreducible characters of $B$ can be divided into $e+1$ classes, of which $e$ classes consist of one nonexceptional character each, and one class consists of the exceptional characters. (If there are no exceptional characters, then $e=p^{a-d+1}-1=p-1$, and there are $e+1$ nonexceptional characters. In this case we choose one of them and treat it below as the exceptional class.) There are exactly $e$ irreducible modular characters of $B$, and each one occurs as a constituent of just those irreducible characters in exactly two of the classes.

We can represent this information with a graph. Let the vertices of the graph correspond to the classes of irreducible characters of $B$, ship.

1 This work was done with the support of a National Science Foundation Fellow- 
and let the edges correspond to the irreducible modular characters. An edge corresponding to the modular character $\phi$ joins the two vertices corresponding to the classes of the characters $\chi_{1}$ and $\chi_{2}$ if and only if $\phi$ is a constituent of $\chi_{1}$ and $\chi_{2}$. The graph turns out to be a tree with $e$ edges and $e+1$ vertices.

If the modular character $\phi$ occurs as a constituent of the irreducible character $\chi$, then it occurs with multiplicity 1 . Thus if we label each edge with the degree of the corresponding modular chatacter, and each vertex with the degree of the characters in the corresponding class, then the value at each vertex is just the sum of the values on the adjacent edges. We have that the degrees of the nonexceptional characters are all $\pm N\left(\bmod p^{a-d+1}\right)$, and the degrees of the exceptional characters are $\pm e N\left(\bmod p^{a-d+1}\right)$, for some $N \not \equiv\left(\bmod p^{a-d+1}\right)$. Furthermore, if two irreducible nonexceptional characters have a modular constituent in common, their degrees have opposite signs $\left(\bmod p^{a-d+1}\right)$. If an irreducible exceptional character and an irreducible nonexceptional character have a modular constituent in common, then their degrees have the same sign $\left(\bmod p^{a-d+1}\right)$, 1.e. they are either $-e N$ and $-N\left(\bmod p^{a-d+1}\right)$ respectively or $+e N$ and $+N$. The values at the vertices are thus $\pm N\left(\bmod p^{a-d+1}\right)$ except for one, which has value $\pm e N\left(\bmod p^{a-d+1}\right)$. Adjacent vertices with values $\pm N\left(\bmod p^{a-d+1}\right)$ have opposite signs $\left(\bmod p^{a-d+1}\right)$. All vertices adjacent to the vertex with value $\pm e N\left(\bmod p^{a-d+1}\right)$ have the same sign as that vertex.

PROOF OF THEOREM. What we must show is that with the values on the vertices satisfying the conditions described above, the values on the edges must be among those listed in the statement of the theorem. Let $v_{0}$ be the vertex with value $\pm e N\left(\bmod p^{\alpha-d+1}\right)$. We may assume that it has the + sign in fact, as we can always achieve this by multiplying the values on all edges and vertices by \pm 1 . Now the signs of the other vertices are also fixed, by the conditions on the signs of adjacent vertices. We claim that the values on the edges are now determined uniquely.

More generally, let $k, m$ be positive integers, and suppose we have a tree with $k$ edges and with values $(\bmod m)$ on the vertices and edges such that at each vertex the sum $(\bmod m)$ of the values of the adjacent edges is congruent $(\bmod m)$ to the value of the vertex. Then the vertex values uniquely determine the edge values. For consider an edge which is adjacent to one of the extreme vertices, i.e. a vertex with only one adjacent edge. The summation condition at the extreme vertex determines the value of the edge. Now delete this edge and extreme vertex from the tree, thereby getting a new tree with 
$k-1$ edges. Give the vertices of this new tree the same values as they had in the original tree except for the vertex which was adjacent to the deleted edge. Let the value for this vertex be the original value minus the value of the deleted edge. If $S$ is a set of values for the edges of the new tree which satisfies the summation conditions at the vertices of the new tree, then $S$ together with the value for the deleted edge satisfies the summation conditions for the vertices of the original tree. Now pick an extreme vertex of the new tree, thereby determining the value of another edge. Continuing in this manner. we see that the values of all the edges are determined.

We now exhibit these uniquely determined values. Let the edges of the tree be $a_{1}, a_{2}, \cdots, a_{\theta}$. For the $i$ th edge let $d_{i}$ be the distance from $v_{0}$ to this edge (i.e. the number of edges in the path from $v_{0}$ to this edge, not counting the edge itself). If this edge is deleted from the tree, some of the vertices are separated from $v_{0}$. Let $s_{i}$ be the number of these. Now set $n_{i} \equiv(-1)^{d_{i} s_{i}} N\left(\bmod p^{a-d+1}\right)$. We claim that the $n_{i}$ are the desired values. Consider any vertex $v \neq v_{0}$. There is one edge at $v$ which is closer to $v_{0}$ than all of the other edges at $v$ by exactly one. The vertices separated from $v_{0}$ by this edge are just those separated by all the other edges at $v$ together with $v$ itself. Thus the sum of the $n_{i}$ at $v$ is $\pm N\left(\bmod p^{a-d+1}\right)$, where the sign agrees with that of the closest edge to $v_{0}$ at $v$. Since adjacent vertices differ in their distances from $v_{0}$ by one, the signs of adjacent vertices (other than $v_{0}$ ) differ. Since the edges adjacent to $v_{0}$ all have positive value, both $v_{0}$ and those vertices adjacent to it have positive values. The value at $v_{0}$ is just $N$ times the sum of the $s_{i}$ for the adjacent edges. But this sum just counts the total number of vertices separated from $v_{0}$ by all of these edges, which is $e$. Thus the value at $v_{0}$ is indeed $e N\left(\bmod p^{a-d+1}\right)$, and the $n_{i}$ are the desired values.

Since $0<s_{i} \leqq e \leqq p-1, i=1,2, \cdots, e$, we see that both the first and second statements of the theorem follow. The third statement is true because the principal character has degree 1 .

\section{REFERENCES}

1. R. Brauer, Investigations on group characters, Ann. of Math. 42 (1941), 936-958.

2. E. C. Dade, Blocks with cyclic defect groups, Ann. of Math. 84 (1966), 20-48.

YALE UNIVERSITY 